\title{
BMJ Open FIRST-line support for Assistance in Breathing in Children (FIRST-ABC): protocol for a multicentre randomised feasibility trial of non-invasive respiratory support in critically ill children
}

Padmanabhan Ramnarayan, ${ }^{1,2}$ Paula Lister, ${ }^{3}$ Troy Dominguez, ${ }^{4}$ Parviz Habibi, ${ }^{2}$ Naomi Edmonds, ${ }^{5}$ Ruth Canter, ${ }^{6}$ Paul Mouncey, ${ }^{6}$ Mark J Peters ${ }^{3,7}$

To cite: Ramnarayan $\mathrm{P}$, Lister $\mathrm{P}$, Dominguez T, et al. FIRSTline support for Assistance in Breathing in Children (FIRST-ABC): protocol for a multicentre randomised feasibility trial of non-invasive respiratory support in critically ill children. BMJ Open 2017;7:e016181. doi:10.1136/ bmjopen-2017-016181

\section{- Prepublication history} for this paper is available online. To view these files please visit the journal online (http://dx.doi.org/10.1136/ bmjopen-2017-016181).

Received 30 January 2017 Revised 17 March 2017 Accepted 24 April 2017

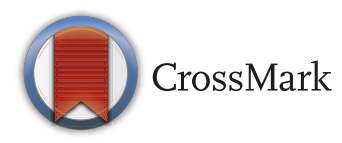

For numbered affiliations see end of article.

Correspondence to

Mark J Peters;

mark.peters@ucl.ac.uk

\section{ABSTRACT}

Introduction Over 18000 children are admitted annually to UK paediatric intensive care units (PICUs), of whom nearly $75 \%$ receive respiratory support (invasive and/ or non-invasive). Continuous positive airway pressure (CPAP) has traditionally been used to provide first-line non-invasive respiratory support (NRS) in PICUs; however, high-flow nasal cannula therapy (HFNC), a novel mode of NRS, has recently gained popularity despite the lack of high-quality trial evidence to support its effectiveness. This feasibility study aims to inform the design and conduct of a future definitive randomised clinical trial (RCT) comparing the two modes of respiratory support.

Methods and analysis We will conduct a threecentre randomised feasibility study over 12 months. Patients admitted to participating PICUs who satisfy eligibility criteria will be recruited to either group $A$ (primary respiratory failure) or group B (postextubation). Consent will be obtained from parents/guardians prior to randomisation in 'planned' group $B$, and deferred in emergency situations (group A and 'rescue' group B). Participants will be randomised (1:1) to either CPAP or HFNC using sealed, opaque envelopes, from a computergenerated randomisation sequence with variable block sizes. The study protocol specifies algorithms for the initiation, maintenance and weaning of HFNC and CPAP. The primary outcomes are related to feasibility, including the number of eligible patients in each group, feasibility of randomising $>50 \%$ of eligible patients and measures of adherence to the treatment protocols. Data will also be collected on patient outcomes (eg, mortality and length of PICU stay) to inform the selection of an appropriate outcome measure in a future RCT. We aim to recruit 120 patients to the study.

Ethics and dissemination Ethical approval was granted by the National Research Ethics Service Committee North East-Tyne\&Wear South (15/NE/0296). Study findings will be disseminated through peer-reviewed journals, national and international conferences.

Trials registration number NCT02612415; pre-results.
Strengths and limitations of this study

- This randomised trial aims to test the feasibility of comparing two modes of non-invasive respiratory support in critically ill children.

- If shown to be feasible, this study will inform the design and conduct of a future definitive randomised trial (RCT) comparing high-flow nasal cannula therapy with continuous positive airway pressure.

- In addition to testing feasibility, data will be collected on several secondary outcomes to inform the selection of an appropriate primary outcome measure for a future RCT.

- As a feasibility study run in three study sites, this study does not however have the power to show clinical effectiveness.

- This feasibility trial will not be assessing the cost effectiveness of the two treatments.

\section{BACKGROUND}

Each year, over 18000 critically ill children are admitted to paediatric intensive care units (PICU) in the UK. ${ }^{1}$ Irrespective of the primary reason for admission, respiratory support is the most common intervention undertaken in PICU; national audit data from the Paediatric Intensive Care Audit Network demonstrate that nearly $75 \%$ of admissions between 2011 and 2013 received either invasive (via an endotracheal tube or tracheostomy) and/or non-invasive respiratory support during their PICU stay. ${ }^{1}$

Although invasive ventilation can be life saving, concerns regarding its complications, such as ventilator-induced lung injury, need for prolonged sedation and nosocomial respiratory tract infections have encouraged the greater adoption of non-invasive 
respiratory support (NRS) techniques in intensive care settings. ${ }^{2-4}$ In critically ill adults and premature newborns, evidence from randomised clinical trials (RCT) supports the early use of NRS to reduce invasive ventilation and improve survival in specific patient subgroups. ${ }^{5-8}$ In critically ill infants and children, there is a dearth of highquality RCT evidence, ${ }^{9}$ yet, the use of NRS has increased over the years in UK PICUs as well as internationally. ${ }^{10}$

Continuous positive airway pressure (CPAP) has traditionally been used as the first-line mode of NRS in the PICU setting, to either avoid intubation and invasive ventilation or to avoid reintubation after extubation, following a spell of invasive ventilation. ${ }^{11}{ }^{12}$ However, the widespread use of CPAP is limited by two main problems: (1) the need for a tight-fitting patient interface such as face mask, hood or nasal prongs to avoid leakage of gas from the ventilator circuit (which frequently causes patient discomfort/agitation as well as nasal and facial pressure sores with prolonged use, leading to treatment failure) and (2) the risk of serious complications such as pneumothorax or pneumomediastinum (which usually necessitates close monitoring and a high level of skilled nursing input).

Over the past decade, a novel mode of NRS, heated-humidified high-flow nasal cannula therapy (HFNC), has rapidly gained popularity despite the absence of RCT evidence to support its effectiveness in the PICU setting. ${ }^{1314}$ The main reason for its increasing use is related to patient comfort and ease of use.$^{15}$ HFNC does not require a tight seal and its patient interface (nasal prongs) is well tolerated by children. There is strong evidence from physiological and observational studies to support the use of HFNC in PICU-HFNC allows the delivery of heated and humidified medical gases to the patient at high-gas flow rates (matching or exceeding the patient's own peak inspiratory flow rate), which has been shown to confer a diverse range of beneficial effects such as reduction of airway resistance, reduction of dead space by nasopharyngeal washout with fresh gas, as well as delivery of positive airway pressure (similar to CPAP). ${ }^{16}{ }^{17}$ Studies in infants and children confirm that HFNC reduces the work of breathing and improves oxygenation and ventilation. ${ }^{18} 19$ In single-centre observational studies, the use of HFNC has been shown to be associated with a dramatic reduction in the rate of intubation and invasive ventilation. ${ }^{20-22}$ However, there have not yet been any RCTs comparing HFNC with other forms of NRS such as CPAP in the PICU setting. ${ }^{23}$

Before an expensive health technology such as HFNC is adopted more widely across the paediatric intensive care setting, it is crucial that evidence from a large pragmatic RCT is urgently available to support its clinical and cost effectiveness, especially since loss of clinical equipoise regarding the risks and benefits of HFNC is already occurring among clinicians. ${ }^{24}$ Prior to a national RCT, however, it is imperative that the feasibility of conducting such an RCT is established. In this paper, we describe the protocol for a multicentre randomised feasibility trial to compare the two most commonly used modes of NRS (CPAP and HFNC) in critically ill children admitted to PICU (V.2.1, 17 March 2016).

\section{STUDY AIM}

The aim of this study is to conduct a feasibility study to inform the design and conduct of a future definitive multicentre RCT comparing two commonly used modes of non-invasive respiratory support (CPAP and HFNC) in the PICU setting.

\section{STUDY OBJECTIVES}

Primary objective: To determine the feasibility of an RCT of HFNC versus CPAP in critically ill children admitted to PICU.

Secondary objectives:

- to determine the rate of intubation and invasive ventilation (or reintubation) in each study arm

- to determine the rate of treatment failure in each study arm

- to assess the safety of the use of CPAP and HFNC

- to assess the physiological effects of CPAP and HFNC

- to assess the effect of CPAP and HFNC on patient outcomes.

\section{STUDY DESIGN AND SETTING}

This is a randomised, controlled, open-label clinical trial comparing HFNC with CPAP as the first-line non-invasive respiratory support modality in critically ill children. Patients will be recruited at three PICUs in London. Together, these PICUs admit around 2500 children annually. The frequency of NRS use is variable on the units (between $15 \%$ and $43 \%$ of admissions) owing to differences in availability of beds for high-dependency care. All three units have access to both modes of NRS (CPAP and HFNC).

\section{INCLUSION CRITERIA}

Pragmatic inclusion criteria will be used. To minimise variation in practice between and within centres, predefined, objective criteria are provided to clinicians to guide the decision on when to start NRS. Eligible patients aged between $>36$ weeks corrected gestational age and $<16$ years will fall into one of two groups:

Group A (step-up)

- Deemed by the treating clinician to require NRS for an acute illness;

- Satisfy one or more of the following criteria:

- hypoxia (oxygen saturation $<92 \%$ in fraction of inspired oxygen $>0.40$, or equivalent)

- acute respiratory acidosis $(\mathrm{pH}<7.3$ with a concomitant partial pressure of carbon dioxide $>6.5 \mathrm{kPa}$ ) 
moderate respiratory distress (use of accessory muscles, subcostal and intercostal recession, tachypnoea for age, grunting).

Group B (step-down)

Deemed by the treating clinician to require NRS following a spell of invasive ventilation, either immediately after extubation as a planned procedure ('planned') or prompted by clinical deterioration within 72 hours after extubation ('rescue').

'Rescue' participants will be required to also satisfy one or more of the following criteria: hypoxia, acute respiratory acidosis or moderate respiratory distress (using the same definitions as above).

\section{EXCLUSION CRITERIA}

Patients will be excluded if they (1) are deemed by the treating clinician to require immediate intubation/invasive ventilation due to severe hypoxia, acidosis and/or respiratory distress, upper airway obstruction or recurrent apnoeas; (2) have a tracheostomy in place; (3) have a pre-existing air-leak syndrome (pneumothorax and/or pneumomediastinum); (4) have midfacial/craniofacial anomalies (unrepaired cleft palate, choanal atresia) or had recent craniofacial surgery; (5) have an agreed limitation of intensive care treatment plan in place ("not for intubation'); (6) have been on domiciliary NRS prior to PICU admission; (7) have been managed on either HFNC and/ or CPAP (or other form of NRS such as bilevel positive airway pressure (BiPAP)) in the preceding 24hours; (8) have been previously recruited to the study during the same PICU admission; (9) cannot be treated with HFNC due to unavailability of appropriate sized nasal prongs or HFNC device; or (10) cannot be treated with CPAP due to unavailability of right size of face mask, prong or other patient interface or CPAP device.

\section{SCREENING FOR ELIGIBILITY}

A Consolidated Standards for Reporting Trials (CONSORT) flow diagram is shown in figure 1. Clinical and/or the research nurse teams will assess patients admitted to study PICUs to identify potentially eligible study participants. Screening procedures will be different for Groups A and B. For Group B, all invasively ventilated patients on the PICU will be screened daily to identify children who are planned

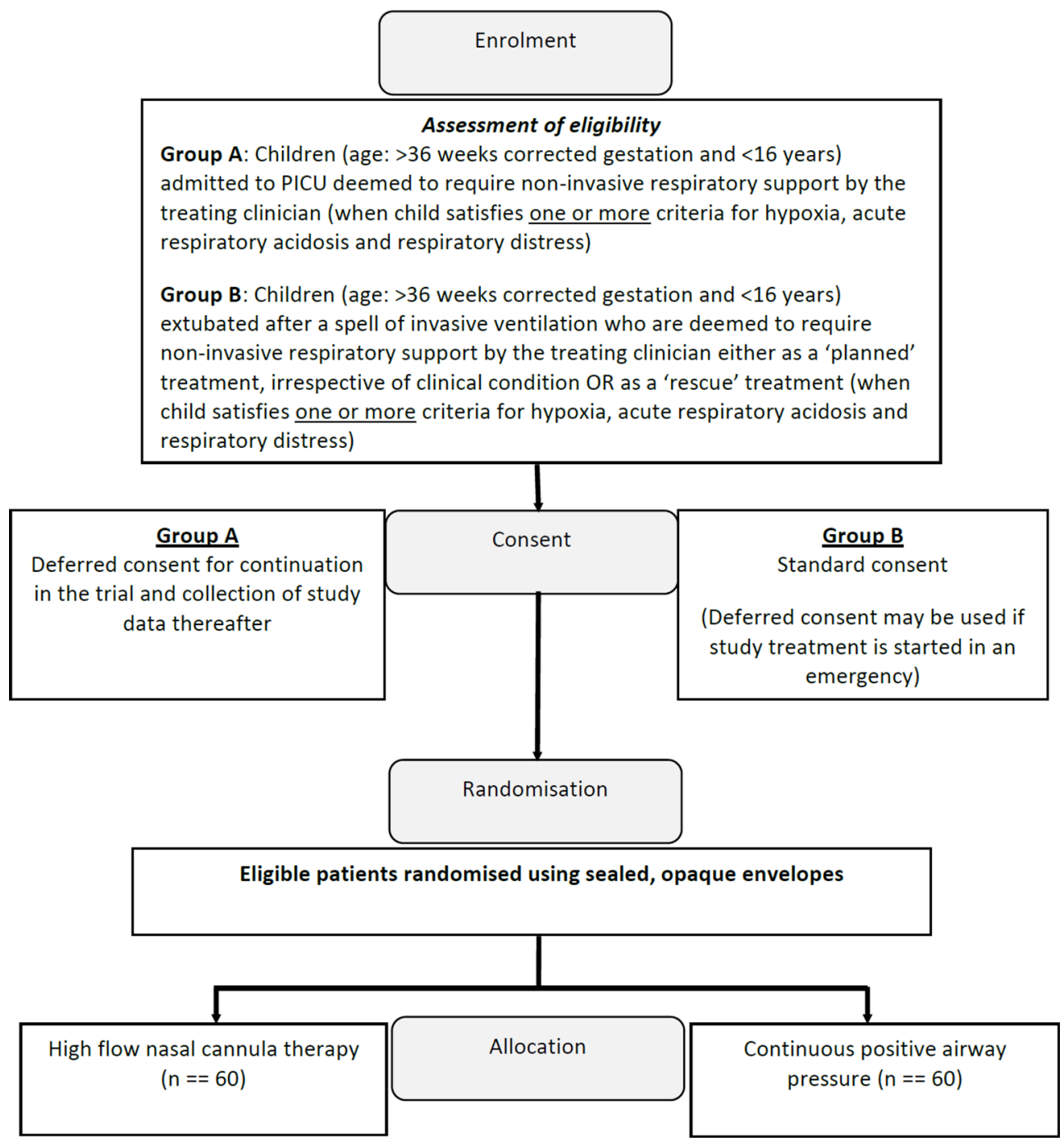

Figure 1 Planned flow of patients through the FIRST-line support for Assistance in Breathing in Children feasibility trial. 


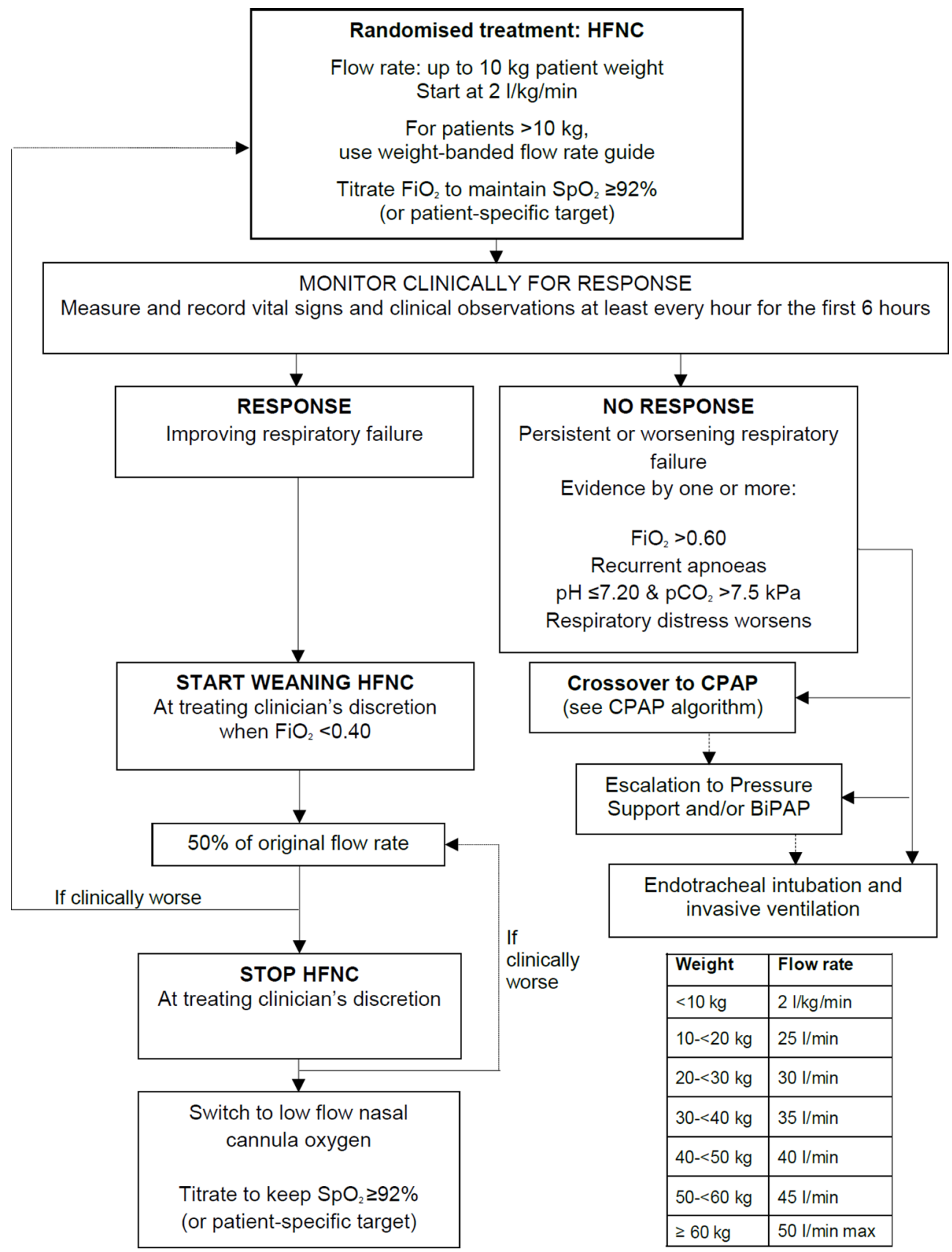

Figure 2 Study algorithm for the management of patients randomised to HFNC. BiPAP, bi-level positive airway pressure; CPAP, continuous positive airway pressure; $\mathrm{FiO}_{2}$, fraction of inspired oxygen; $\mathrm{HFNC}$, high-flow nasal cannula; $\mathrm{PaO}_{2}$, partial pressure of arterial oxygen; $\mathrm{pCO}_{2}$, partial pressure of carbon dioxide.

for extubation. The treating clinician will be approached to establish whether the patient would be placed on NRS immediately after extubation irrespective of clinical condition ('planned'), or whether NRS would only be used as a 'rescue' treatment after extubation. A screening log of all patients who fulfil inclusion criteria but meet exclusion criteria, as well as a log of eligible patients who are not recruited to the study, will be maintained.

\section{PATIENT RECRUITMENT AND CONSENT}

A mixed model of consent will be utilised (prospective and deferred) appropriate to the nature of the clinical situation (planned initiation of NRS or emergency initiation of NRS). Informed consent will be supported by providing information to parents/guardians at different stages of the patient pathway.

Group A: Patients requiring NRS as a 'step-up' treatment will most often need this started in a life-threatening emergency, where any delay in commencing treatment will be detrimental, making any attempt to obtain fully informed consent from parents/guardians during an emergency inappropriate and cause additional stress to families who are already distressed by their child's illness. Therefore, consent in this situation will be deferred. 


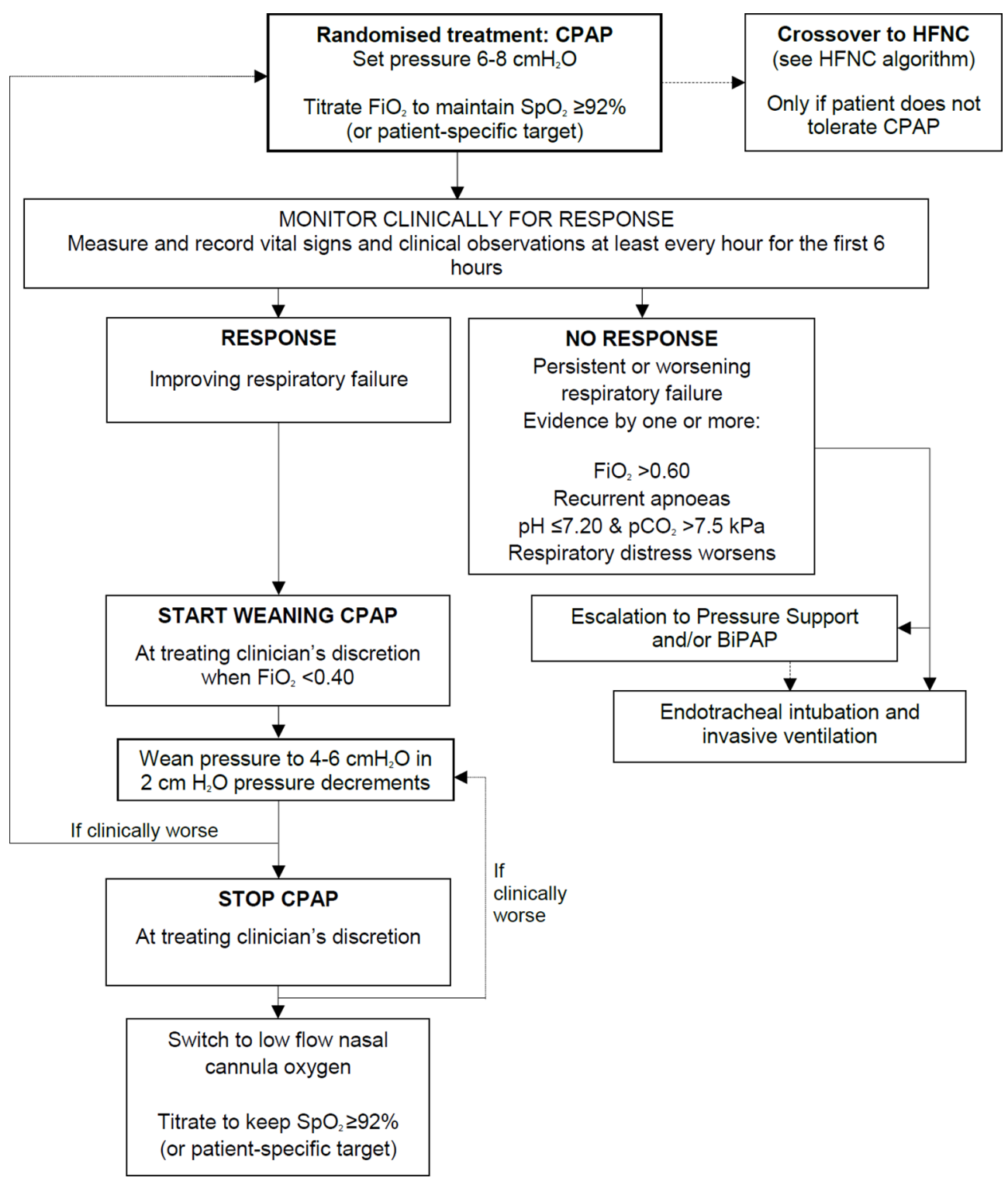

Figure 3 Study algorithm for the management of patients randomised to CPAP. BiPAP, bi-level positive airway pressure; CPAP, continuous positive airway pressure; $\mathrm{FiO}_{2}$, fraction of inspired oxygen; $\mathrm{HFNC}$, high-flow nasal cannula; $\mathrm{pCO}_{2}$, partial pressure of carbon dioxide; $\mathrm{SpO}_{2}$, normal blood oxygen saturation level.

Once notified of the recruitment of a patient to the study, the clinical/research nurse team will approach the parents/guardians as soon as practically possible after randomisation (usually within $24-48$ hours) to discuss the study, provide written information and seek informed consent. Consent will be sought for continuation in the trial and for data collection from routine medical records. Both modes of NRS (CPAP and HFNC) are relatively safe, commonly used in clinical practice and in practice only determined by individual clinician preferences.

Group B: Patients requiring NRS as a 'step-down' treatment will be receiving invasive ventilation on PICU. Therefore, there will be sufficient time during which the clinical/research nurse team can discuss the study and provide detailed written information to the parents/guardians. Following this discussion, if parents/guardians refuse to participate in the research, no further involvement in the study will be considered. If NRS is 'planned' following extubation by the treating clinician, written consent will be obtained from parents/guardians by the clinical/research nurse team before randomisation. If NRS is initiated as a 'rescue' intervention following extubation, written consent may be deferred, depending on parental availability and the emergency nature of the situation. This was left at the discretion of the clinical team.

Due to the use of deferred consent, there may be rare situations where the patient is either: (1) discharged from hospital prior to consent being obtained from the parents/guardians or (2) the patient dies prior to consent being sought. In the former situation, we will aim to obtain postal consent as soon as possible after discharge, by sending parents/guardians study 
Table 1 Schedule of events

\begin{tabular}{|c|c|c|c|c|c|c|}
\hline & $\begin{array}{l}\text { PICU admission } \\
\text { OR } \\
\text { prior to extubation }\end{array}$ & Study entry & $\begin{array}{l}\text { Hourly for first } \\
6 \text { hours }\end{array}$ & $\begin{array}{l}\text { At } 12,24,36,48 \\
\text { and } 72 \text { hours until } \\
\text { end of treatment }\end{array}$ & $\begin{array}{l}\text { Hospital } \\
\text { discharge } \\
\text { (or death) }\end{array}$ & At day 28 \\
\hline Screening for eligibility & $x$ & & & & & \\
\hline $\begin{array}{l}\text { Informed consent and } \\
\text { consent questionnaire }\end{array}$ & & $x$ & & $X^{*}$ & & \\
\hline Randomisation & & $x$ & & & & \\
\hline Physiology & & & $x$ & $x$ & & \\
\hline COMFORT score & & & $x$ & $x$ & & \\
\hline $\begin{array}{l}\text { PSS:PICU Questionnaire } \\
\text { (at } 24 \text { hours only) }\end{array}$ & & & & $x$ & & \\
\hline
\end{tabular}

${ }^{*}$ Deferred consent will be sought as soon as practically possible, usually within 48 hours.

CPAP, continuous positive airway pressure; HFNC, high-flow nasal cannula; PSS, Parental Stressor Scale; PICU, Paediatric Intensive Care Unit.

information on two separate occasions 4 weeks apart. If no consent form is received within 4 weeks of receipt of the letter, then the participants' data will be included in the study unless the family notify the site research team otherwise. In the latter situation, the parents/guardians will not be informed of their child's involvement in the trial as this may cause unnecessary and avoidable distress. Data up to the patient's death will still be collected and used as part of the study, as there may be a risk of bias if this was removed.

If prior consent is not provided for patients in group B, they will not be randomised to the trial. A minimal dataset will be collected for each patient approached but not randomised including study site, date/time approached and reason for non-consent. If deferred consent is not provided for patients in group A following randomisation, no further data will be collected from the child and the child will be recorded as not consented. Data collected up to the point of parental refusal of consent will be used. A minimal dataset will be collected for each patient randomised but not consented including study site, date/time randomised, randomised intervention (including whether started on assigned intervention or not) and reason for non-consent.

\section{RANDOMISATION AND BLINDING}

Randomisation will be performed as soon as possible after identifying the child as being eligible for the study (no later than 24 hours). Prerandomisation stratification will be by group (A or B) and by study site. Eligible patients will be randomised on a $1: 1$ basis to either CPAP or HFNC using sealed, opaque envelopes available at each centre. The randomisation sequence will be computer generated with variable block sizes to strengthen allocation concealment. The intervention in this study cannot be blinded, since both treatments
(CPAP and HFNC) are already used in practice and recognisable by clinical staff. Study investigators, including those performing the final analysis, will be blinded to the allocation.

\section{STUDY INTERVENTION}

A commercially available and Conformité Européene (CE)-marked HFNG device will be used to deliver a prescribed gas flow rate for the duration that the patient needs NRS. The study protocol specifies clinical criteria and procedures for the initiation, maintenance and weaning of HFNC (see figure 2 for study algorithm). As per current practice, clinicians in the study will be able to stop HFNC and crossover to CPAP if clinically deemed necessary. Prespecified objective criteria will be provided in the study protocol as a guide for clinicians considering crossover from HFNC to CPAP to identify non-responders to HFNC. Reasons for crossover will be recorded. Crossover patients will remain in the study and continue to be monitored until they are off respiratory support.

\section{CONTROL}

A commercially available and CE-marked CPAP device will be used to provide a set expiratory pressure of $6-8 \mathrm{~cm}$ $\mathrm{H}_{2} \mathrm{O}$ for the duration that the infant needs NRS. The study protocol specifies clinical criteria and procedures for the initiation, maintenance and weaning of CPAP (see figure 3 for study algorithm). As per current practice, clinicians will be able to stop CPAP and crossover to HFNC only if the patient has significant discomfort/ intolerance to the CPAP. Crossover patients will remain in the study and continue to be monitored until they are off respiratory support. 


\section{CLINICAL MANAGEMENT}

Recruited patients will be treated as per the study protocol with respect to the provision of NRS. Due to the pragmatic nature of the trial, all other treatment in both groups will be as per standard practice at the study sites. Infants who fail to improve on CPAP or HFNC may be escalated to other non-invasive modes of ventilation such as BiPAP or pressure support (before intubation and ventilation) as per the treating clinician's discretion.

\section{DATA COLLECTION}

A full schedule of assessments is provided in table 1 . Patient demographics will be collected at randomisation (age, gender, primary reason for PICU admission, comorbidities). Routine clinical observations such as normal blood oxygen saturation level, fraction of inspired oxygen, potential of hydrogen, partial pressure of carbon dioxide, heart rate, respiratory rate and clinical signs of respiratory distress, a modified COMFORT score to indicate patient tolerance to the treatment (excluding the respiratory component) and use of sedative agents to improve tolerance to the treatment will be collected at the start of the randomised treatment and assessed on an hourly basis for the first 6 hours, then at 12, 24, 36, 48 and 72 hours until the end of the assigned treatment (or crossover, escalation or intubation/ventilation). A consent questionnaire used in other paediatric RCTs using deferred consent (CATCH trial, ISRCTN34884569 and EcLiPSE trial, ISRCTN22567894) will be administered to all parents/ guardians (see online supplementary material 1) irrespective of whether consent is provided to participate in the trial or not. ${ }^{25-27}$ Parents/guardians will also be asked to complete a validated instrument, the Parental Stressor Scale: Paediatric Intensive Care Unit (PSS:PICU) questionnaire, around 24 hours after initiation of NRS to assess parental stress (see online supplementary material 2). ${ }^{28}$ Adverse events will be collected and assessed for duration, causality, expectedness, seriousness and severity.

\section{DATA MANAGEMENT AND MONITORING}

Study data, including serious adverse events, will be collected and managed using Research Electronic Data Capture (REDCap) electronic data capture tools managed by the Intensive Care National Audit and Research Centre (ICNARC) Clinical Trials Unit (CTU). ${ }^{29}$ REDCap is a secure, web-based application designed to support data capture for research studies, providing (1) an intuitive interface for validated data entry, (2) audit trails for tracking data manipulation and export procedures, (3) automated export procedures for seamless data downloads to common statistical packages and (4) procedures for importing data from external sources. Study participants will be identified by a unique study specific number. The name and any other identifying detail will not be included in any study data electronic file. Research assistants and statisticians will carry out periodic data quality checks and clarify data errors with research sites.
This is a low-risk trial and major safety data are not anticipated. Serious adverse events (SAEs) will be reported to the ICNARC CTU unless otherwise defined in the protocol. Trends in SAEs will be monitored by the CTU, and unexpected SAEs will be notified to the Sponsor. The ICNARC CTU will conduct at least one monitoring visit to participating sites during the course of the trial. No formal data monitoring and ethics committee will be established since this is a feasibility study.

\section{OUTCOME MEASURES}

The primary outcomes are related to the primary study objective, namely determining the feasibility of a future RCT: (1) number of eligible patients in group A (step-up treatment) and group B (step-down treatment); (2) feasibility of randomising at least $50 \%$ of eligible patients; (3) acceptability of using a mixed model of consent (prospective and deferred); (4) adherence to the study protocol in terms of initiation, maintenance and weaning of the study treatments; (5) use of a modified COMFORT score to assess patient tolerance; and (6) use of the PSS:PICU questionnaire to measure parental stress 24 hours after the initiation of the treatment. Data will be collected on patient outcomes to inform the choice of an appropriate outcome measure for the definitive trial (rate of intubation, rate of treatment failure, rate of crossover/ escalation, length of stay on PICU and in hospital, length of invasive and non-invasive ventilation and mortality in PICU and at hospital discharge).

\section{DATA ANALYSIS}

We will use intention-to-treat analysis to perform any comparisons between the groups, although as a feasibility study, this is not the main aim of the trial. Since crossover will be allowed, we will also perform a per-protocol analysis. We will calculate the rate of recruitment (number of patients randomised/number of eligible patients) for each group, the consent rate (number of patients consented/number of patients approached for consent) for prospective and deferred consent, rate of crossover (number of patients crossed over to the other treatment/ number of patients randomised to the treatment) for each arm, the rate of intubation (number of patients needing intubation/number of patients randomised to the treatment) for each arm and the frequency of serious adverse events occurring in each treatment arm. As a feasibility study, no formal sample size calculations have been performed. Based on analysis of audit data, we expect around 250 eligible patients over the 6 -month period at the three sites. Assuming a 50\% recruitment rate, we will have recruited 120 study patients (around 40 patients in group A). Data from the literature suggests a $20 \%$ rate of intubation for group A (ie, we expect to see eight intubation events) and a 10\% rate of reintubation for group B (ie, we expect to see eight reintubation events). 


\section{ETHICAL APPROVAL AND REGULATORY CONSIDERATIONS}

Ethical approval was provided by the National Research Ethics Service Committee North East-Tyne and Wear South (ref: 15/NE/0296). Approval was obtained from all participating sites' research and development departments prior to the study initiation. The study protocol, patient information sheets, informed consent forms and other study-related documents were reviewed and approved by the Sponsor and Research Ethics Committee with respect to scientific content and compliance with applicable research regulations involving human subjects. Since the trial involves the use of CE-marked medical devices employed for their intended purpose, it is not considered to be a clinical investigation under the Medical Devices Regulations 2002, nor does it fall within the remit of the Medicines for Human Use (Clinical Trials) Regulations 2004. The study will comply with the Data Protection Act, 1998.

\section{DISSEMINATION STRATEGY}

The results of the study will be reported and disseminated via peer-reviewed scientific journals, conference presentations and written feedback to patient support groups.

\section{DISCUSSION}

Although CPAP has been used as a mode of non-invasive respiratory support for over two decades, its use in critically ill children is not supported by clinical trial evidence. ${ }^{9}$ HFNC therapy has recently become a popular alternative to CPAP since it is better tolerated by patients and is easy to use. ${ }^{30} 31$ Although observational studies indicate that the use of HFNC may reduce the need for intubation/ invasive ventilation, there have been no RCTs comparing the clinical and cost-effectiveness of HFNC with CPAP in critically ill children. ${ }^{20-22} 32$

The design and conduct of a definitive RCT comparing HFNC and CPAP in critically ill children potentially involves several challenges. First, since the use of HFNC has superseded the use of CPAP in many paediatric settings, there is a risk that some clinicians (and/or parents) may be reluctant to randomise patients to one or the other treatment. Second, the lack of robust evidence to guide clinicians on when and which patients to select for NRS has resulted in variability in clinical practice; this makes it important that any study algorithms used for the initiation, maintenance and weaning of HFNC/CPAP are acceptable to clinicians and practical to use. Third, since RCTs involving the use of HFNC in premature newborns and adults have studied a range of clinical outcomes with varying results, ${ }^{33-36}$ an important consideration for PICU patients is the choice of an appropriate and clinically relevant outcome measure. For all these reasons, it is crucial that a future definitive RCT is preceded by a feasibility trial. Findings from the FIRST-line support for Assistance in Breathing in Children feasibility trial will be used to inform the design and conduct of a future definitive trial.
Author affiliations

${ }^{1}$ Children's Acute Transport Service, Critical Care Division, Great Ormond Street Hospital NHS Foundation Trust, London, UK

${ }^{2}$ Paediatric Intensive Care Unit, St Mary's Hospital, Imperial College Healthcare NHS Trust, London, UK

${ }^{3}$ Paediatric and Neonatal Intensive Care Unit, Critical Care Division, Great Ormond Street Hospital NHS Foundation Trust, London, UK

${ }^{4}$ Cardiac Intensive Care Unit, Critical Care Division, Great Ormond Street Hospital NHS Foundation Trust, London, UK

${ }^{5}$ Paediatric Critical Care Unit, Royal London Hospital, Barts Health NHS Trust, London, UK

${ }^{6}$ Clinical Trials Unit, Intensive Care National Audit and Research Centre (ICNARC), Napier House, High Holborn, London, UK

${ }^{7}$ Respiratory, Critical Care and Anaesthesia Section, Infection, Immunity and Inflammation Programme, UCL Great Ormond Street Institute of Child Health, London, UK

Acknowledgements The authors would like to thank the research staff at participating sites for patient recruitment and data collection. Great Ormond Street Hospital: Lauran O'Neill, Eugenia Abaleke, Katie Hardy, Francesca Carr, Amy Jones; St Mary's Hospital: Leena Mathew, Sarah Darnell, Amina Abdulla; Royal London Hospital: Kevin Goddard, Bessie Crone. The authors would also like to thank Nick Hudson (Data Manager) and Steve Saunders (Research Assistant) from ICNARC CTU for study data management. The authors would like to acknowledge the loan of six additional Airvo machines (high-flow nasal cannula therapy devices) from Fisher and Paykel Healthcare UK to Great Ormond Street Hospital and St Mary's Hospital for a period of 6 months to support the study.

Contributors PR is the chief investigator of the study. He conceived the study and drafted the manuscript. PL, TD, PH, NE and MJP are study coapplicants and site principal investigators and made a significant contribution to the design and conduct of the study. RC is Study Manager at the ICNARC CTU responsible for day-to-day study management. PM is Senior Trial Manager at ICNARC CTU. Both have contributed to the design, inception and acquisition of data for the study. All authors commented critically on the manuscript and read and approved the final manuscript.

Funding The FIRST-ABC feasibility study was funded by the Great Ormond Street Hospital Children's Charity (ref: V0316). The study is supported by the National Institute for Health Research Biomedical Research Centre at Great Ormond Street Hospital for Children NHS Foundation Trust and UCL Great Ormond Street Institute of Child Health. The trial sponsor is Great Ormond Street Hospital NHS Foundation Trust, Joint R\&D Office GOSH/ICH, 30 Guilford Street, London WC1N 1EH, United Kingdom (email: Research.Governance@gosh. nhs.uk). The Clinical Trials Unit for the study was the ICNARC CTU. The views expressed in this publication are those of the authors and not necessarily of the funder or the sponsor.

Competing interests None declared.

Patient consent Not obtained.

Ethics approval Ethical approval for the study was provided by the National Research Ethics Committee (NRES) North East-Tyne and Wear South (ref:15/ $\mathrm{NE}$ /0296). Management approvals were obtained from all study sites.

Provenance and peer review Not commissioned; externally peer reviewed.

Open Access This is an Open Access article distributed in accordance with the Creative Commons Attribution Non Commercial (CC BY-NC 4.0) license, which permits others to distribute, remix, adapt, build upon this work non-commercially, and license their derivative works on different terms, provided the original work is properly cited and the use is non-commercial. See: http://creativecommons.org/ licenses/by-nc/4.0/

C Article author(s) (or their employer(s) unless otherwise stated in the text of the article) 2017. All rights reserved. No commercial use is permitted unless otherwise expressly granted.

\section{REFERENCES}

1. Paediatric Intensive Care Audit Network(PICANet). 2014 Annual Report - Tables and Figures:Universities of Leeds and Leicester, 2014. 
2. Dohna-Schwake C, Stehling F, Tschiedel E, et al. Non-invasive ventilation on a pediatric intensive care unit: feasibility, efficacy, and predictors of success. Pediatr Pulmonol 2011;46:1114-20.

3. Keenan SP, Sinuff T, Burns KE, et al. Clinical practice guidelines for the use of noninvasive positive-pressure ventilation and noninvasive continuous positive airway pressure in the acute care setting. CMAJ 2011;183:E195-E214.

4. Burns KE, Meade MO, Premji A, et al. Noninvasive ventilation as a weaning strategy for mechanical ventilation in adults with respiratory failure: a Cochrane systematic review. CMAJ 2014;186:E112-E122.

5. Nava S, Carbone G, DiBattista N, et al. Noninvasive ventilation in cardiogenic pulmonary edema: a multicenter randomized trial. Am J Respir Crit Care Med 2003;168:1432-7.

6. Antonelli M, Conti G, Rocco M, et al. A comparison of noninvasive positive-pressure ventilation and conventional mechanical ventilation in patients with acute respiratory failure. $N$ Engl J Med 1998;339:429-35.

7. Ho JJ, Subramaniam P, Davis PG. Continuous distending pressure for respiratory distress in preterm infants. Cochrane Database Syst Rev 2015;7:CD002271.

8. Roehr CC, Proquitté $\mathrm{H}$, Hammer $\mathrm{H}$, et al. Positive effects of early continuous positive airway pressure on pulmonary function in extremely premature infants: results of a subgroup analysis of the COIN trial. Arch Dis Child Fetal Neonatal Ed 2011;96:F371-F373.

9. Shah PS, Ohlsson A, Shah JP. Continuous negative extrathoracic pressure or continuous positive airway pressure compared to conventional ventilation for acute hypoxaemic respiratory failure in children. Cochrane Database Syst Rev 2013;11:CD003699.

10. Ducharme-Crevier L, Essouri S, Emeriaud G. Noninvasive ventilation in pediatric intensive care: from a promising to an established therapy, but for whom, when, why, and how? Pediatr Crit Care Med 2015;16:481-2.

11. Donlan M, Fontela PS, Puligandla PS. Use of continuous positive airway pressure (CPAP) in acute viral bronchiolitis: a systematic review. Pediatr Pulmonol 2011;46:736-46.

12. Cam BV, Tuan DT, Fonsmark L, et al. Randomized comparison of oxygen mask treatment vs. nasal continuous positive airway pressure in dengue shock syndrome with acute respiratory failure. $J$ Trop Pediatr 2002;48:335-9.

13. Lee JH, Rehder KJ, Williford L, et al. Use of high flow nasal Cannula in critically ill infants, children, and adults: a critical review of the literature. Intensive Care Med 2013:39:247-57.

14. Spentzas T, Minarik M, Patters AB, et al. Children with respiratory distress treated with high-flow nasal Cannula. J Intensive Care Med 2009;24:323-8.

15. Klingenberg C, Pettersen M, Hansen EA, et al. Patient comfort during treatment with heated humidified high flow nasal cannulae versus nasal continuous positive airway pressure: a randomised cross-over trial. Arch Dis Child Fetal Neonatal Ed 2014;99:F134-F137.

16. Dysart KC. Physiologic basis for nasal continuous positive Airway pressure, heated and humidified High-Flow nasal Cannula, and nasal ventilation. Clin Perinatol 2016;43:621-31.

17. Dysart K, Miller TL, Wolfson MR, et al. Research in high flow therapy: mechanisms of action. Respir Med 2009;103:1400-5.

18. Pham TM, O'Malley L, Mayfield S, et al. The effect of high flow nasal Cannula therapy on the work of breathing in infants with bronchiolitis. Pediatr Pulmonol 2015;50:713-20.
19. Hough JL, Pham TM, Schibler A. Physiologic effect of high-flow nasal cannula in infants with bronchiolitis. Pediatr Crit Care Med 2014;15:e214-e219.

20. Schibler A, Pham TM, Dunster KR, et al. Reduced intubation rates for infants after introduction of high-flow nasal prong oxygen delivery. Intensive Care Med 2011;37:847-52.

21. McKiernan C, Chua LC, Visintainer PF, et al. High flow nasal cannulae therapy in infants with bronchiolitis. J Pediatr 2010;156:634-8.

22. Kawaguchi A, Yasui $Y$, deCaen A, et al. The clinical impact of heated humidified High-Flow nasal Cannula on Pediatric respiratory distress. Pediatr Crit Care Med 2017;18:112-9.

23. Mayfield S, Jauncey-Cooke J, Hough JL, et al. High-flow nasal cannula therapy for respiratory support in children. Cochrane Database Syst Rev 2014;3:CD009850.

24. Turnham H, Agbeko RS, Furness J, et al. Non-invasive respiratory support for infants with bronchiolitis: a national survey of practice. BMC Pediatr 2017;17:20.

25. Harron K, Woolfall K, Dwan K, et al. Deferred consent for randomized controlled trials in emergency care settings. Pediatrics 2015;136:e13 $16-\mathrm{e} 1322$.

26. Woolfall K, Frith L, Gamble C, et al. CONNECT advisory group. How parents and practitioners experience research without prior consent (deferred consent) for emergency research involving children with life threatening conditions: a mixed method study. BMJ Open 2015;5:e008522.

27. Jansen-van der Weide MC, Caldwell PH, Young B, et al. Clinical trial decisions in Difficult Circumstances: parental Consent under Time pressure. Pediatrics 2015;136:e983-e992.

28. Carter MC, Miles MS. The Parental Stressor Scale: pediatric intensive care unit. Matern Child Nurs J 1989;18:187-98.

29. Harris PA, Taylor R, Thielke R, et al. Research electronic data capture (REDCap) - a metadata-driven methodology and workflow process for providing translational research informatics support. J Biomed Inform 2009:42:377-81.

30. Ramnarayan P, Schibler A. Glass half empty or half full? The story of high-flow nasal Cannula therapy in critically ill children. Intensive Care Med 2017:43:246-9.

31. Jones AJ, Mathew S, Wong SW, et al. A regional audit of high-flow nasal Cannula therapy use for bronchiolitis in London district general hospitals. Arch Dis Child 2017;102:296-7.

32. Abboud PA, Roth PJ, Skiles CL, et al. Predictors of failure in infants with viral bronchiolitis treated with high-flow, high-humidity nasal cannula therapy*. Pediatr Crit Care Med 2012;13:e343-e349.

33. Manley BJ, Owen LS, Davis PG. High-flow nasal cannulae in very preterm infants after extubation. N Engl J Med 2014;370:385-6.

34. Roberts CT, Owen LS, Manley BJ, et al. A multicentre, randomised controlled, non-inferiority trial, comparing high flow therapy with nasal continuous positive airway pressure as primary support for preterm infants with respiratory distress (the HIPSTER trial): study protocol. BMJ Open 2015;5:e008483.

35. Frat JP, Thille AW, Mercat A, et al. High-flow oxygen through nasal cannula in acute hypoxemic respiratory failure. $N$ Engl $J$ Med 2015;372:2185-96.

36. Milési C, Essouri S, Pouyau R, et al. High flow nasal cannula (HFNC) versus nasal continuous positive airway pressure (nCPAP) for the initial respiratory management of acute viral bronchiolitis in young infants: a multicenter randomized controlled trial (TRAMONTANE study). Intensive Care Med 2017;43:209-16. 